\title{
Enabling EUV Resist Research at the 1x and Smaller Regime
}

\author{
Patrick Naulleau ${ }^{1}$, Christopher Anderson ${ }^{1}$, Weilun Chao ${ }^{1}$, Kenneth Goldberg ${ }^{1}$, \\ Antoine Wojdyla ${ }^{1}$, Suchit Bhattarai ${ }^{2}$, Andrew Neureuther ${ }^{2}$, Frank Goodwin ${ }^{3}$, Mark Neisser ${ }^{3}$ \\ ${ }^{1}$ Center for X-ray Optics, Lawrence Berkeley National Laboratory, Berkeley, CA, USA \\ ${ }^{2}$ EECS, University of California, Berkeley, CA, USA \\ ${ }^{3}$ SEMATECH, Albany, NY, USA
}

\begin{abstract}
With the slipping of the insertion node for extreme ultraviolet lithography, demands on resist resolution have increased further stressing sensitivity requirements. A variety of resists, both chemically amplified and not, have been developed meeting resolution needs, but falling short on sensitivity and line-width roughness (LWR). Note that resolution is an absolute mandatory requirement, the true tradeoff that must be considered is between sensitivity and contact hole printing is a crucial application for extreme ultraviolet lithography and is particularly challenged by resist sensitivity due to inherent inefficiencies in darkfield contact printing. Checkerboard strong phase shift masks have the potential to alleviate this problem through a $4 \times$ increase in optical efficiency. The feasibility of this method is demonstrated using the SEMATECH-Berkeley Microfield Exposure Tool pseudo phase shift mask configuration and preliminary results are provided on the fabrication of an etched multilayer checkerboard phase shift mask.
\end{abstract}

Keywords: photoresist, extreme ultraviolet, shot noise, phase-shift mask

\section{Introduction}

With the slipping of the insertion node for extreme ultraviolet (EUV) lithography, demands on EUV resist resolution have increased further stressing sensitivity requirements. A variety of resists, both chemically amplified and not, have been developed meeting resolution needs, but falling short on sensitivity and line-width roughness (LWR). Noting that resolution is an absolute mandatory requirement for EUV resists and cannot be relaxed, the true tradeoff that must be considered is between sensitivity and LWR. In this manuscript we present the concept of dose-to-requirement (DTR) which estimates the intrinsic sensitivity limit of the material. We then use this concept to look at a variety of resists.

Given the extreme pressures on resist sensitivity and the fact that contact-hole printing is expected to be the primary early application of EUV lithography, we continue our exploration of ultrahigh efficiency checkerboard phase shift masks [1,2]. In this manuscript we describe the fabrication of a checkerboard phase shift mask, and present both characterization and imaging throughput results using an EUV aerial image microscope demonstrating throughput gains of greater than $4 \mathrm{X}$.

\section{Dose-to-requirement (DTR)}

Given the inflexible requirement for resolution in the resolution-LRW-sensitivity (RLS) tradeoff [3], the only practical terms available to trade off are LWR and sensitivity. This more limited tradeoff space can be better represented by quantifying the intrinsic sensitivity limit of the resist. The first step in this process is estimating the photon-limited LWR based on the measured sensitivity, resist blur, and absorptivity. This LWR is then compared to the target LWR to estimate the sensitivity required in order to achieve the 
target LWR assuming fixed absorptivity and blur. We refer to this sensitivity as DTR which can be expressed as

$$
\mathrm{DTR}=S_{m}\left(L_{p} / L_{t}\right)^{2}
$$

where $S_{m}$ is the measured sensitivity, $L_{m}$ is the estimated photon LWR, and $L_{p}$ is the target LWR. The expression in Eq. [1] comes directly from the theoretical expression of the RLS tradeoff [3].

The resulting number is an estimate of the best dose that could be achieved in the resist while meeting the LWR requirements assuming the material LWR terms [2] are ignored. We should note that ignoring the material term is certainly not a trivial assumption given that in most cases the estimated material LWR on its own remains larger than the target LWR. That being said, the material term is not directly affected by dose and thus not considered in the DTR expression as opposed to the strictly measurement based Z-factor metric [4].

The process of estimating the photon-limited LWR is achieved using the stochastic Multivariate Poisson Propagation Model (MPPM) [2,5-7]. Some of the key variables in the model include the absorptivity and sensitivity, which can both be directly measured, and the deprotection blur which is estimated based on the correlation length of the measured LWR [2].

To demonstrate the efficacy of the MPPM model, we compare the model-produced deprotection point spread function (PSF) to measured resist blur using the contact-hole exposure latitude method [8] for a commercial chemically amplified resist. Figure 1 shows the model PSF which is generated by seeding the model with an aerial image comprised of light concentrated into a single pixel which is on the order of $1-\mathrm{nm}$ in size. Characterization of the resulting PSF yields a full-width-at-half-maximum width of $9 \mathrm{~nm}$. We then compare this to the contact-hole exposure latitude method results (Fig. 2) which again yields an estimated blur of $9 \mathrm{~nm}$. So using LWR correlation length characterization on line-space prints to predict the deprotection blur term in the model, we were able to accurately model the exposure latitude performance of an independent print of contact holes.
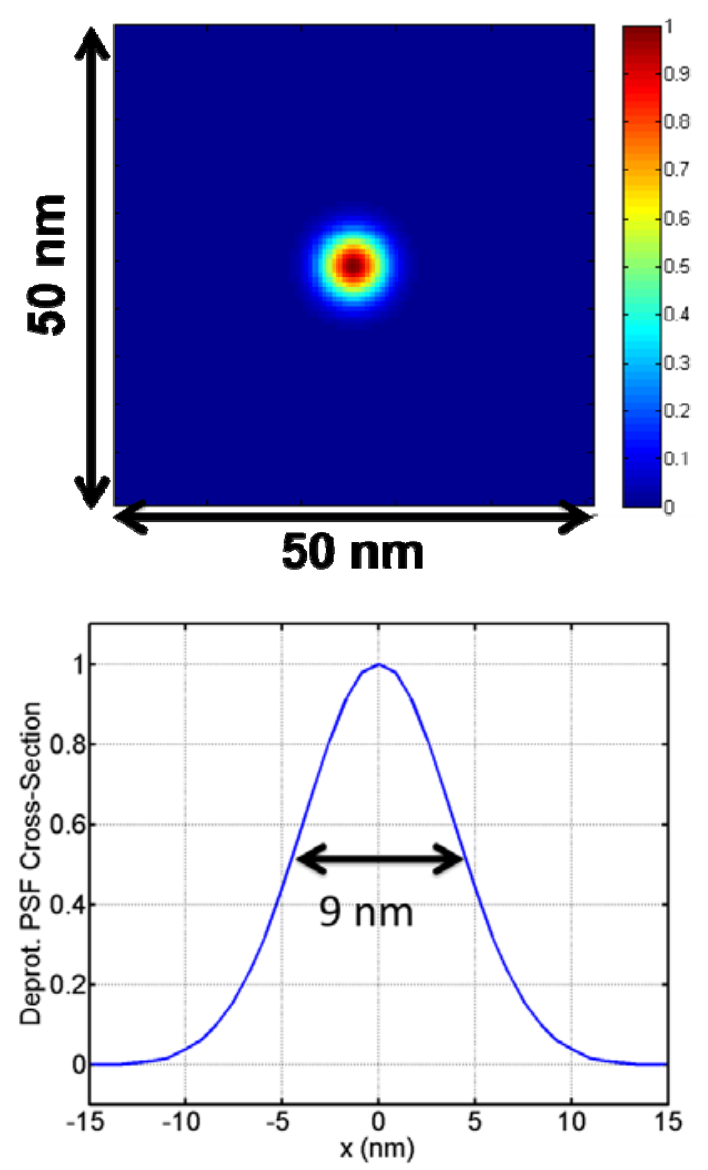

Figure 1. Modeled deprotection PSF for an example chemically amplified resist.

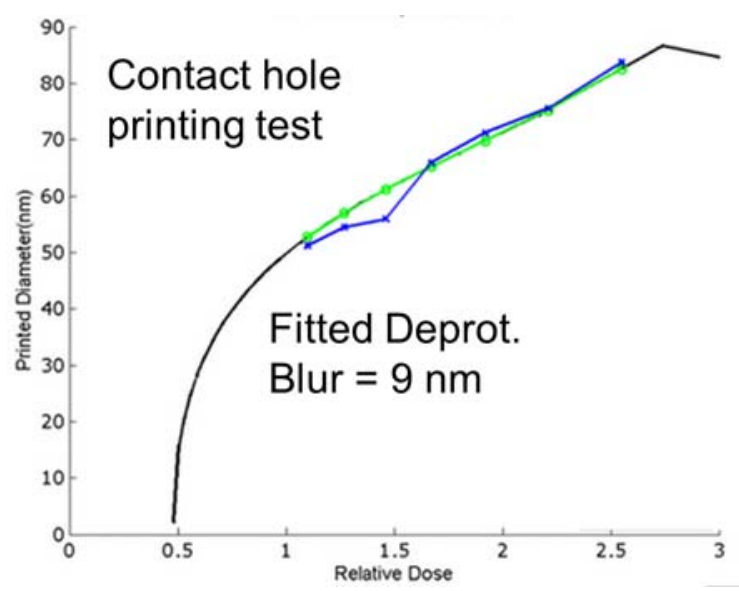

Figure 2. Measured deprotection blur based on independent contact hole patterning test. 
We further compare the MPPM predicted 50 -nm line-space patterning results to the experimental results based on sensitivity, LWR, and exposure latitude. In this case the model is configured to enable all the material stochastic terms in addition to the photon term and the results are shown in Fig. 3.

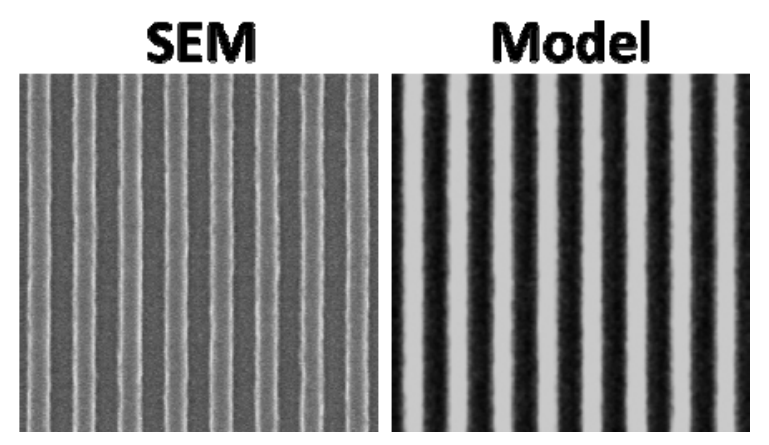

\begin{tabular}{|l|l|l|}
\hline Parameter & Experiment & Model \\
\hline Dose to size & $41.3 \mathrm{~mJ} / \mathrm{cm}^{2}$ & $40.6 \mathrm{~mJ} / \mathrm{cm}^{2}$ \\
\hline Exp. Lat. & 0.279 & 0.312 \\
\hline LER & $2.8 \pm 0.4 \mathrm{~nm}$ & $2.5 \pm 0.2 \mathrm{~nm}$ \\
\hline
\end{tabular}

Figure 3. Direct comparison of modeled and measured 50-nm lines space patterning results.

Next we use the MPPM model disabling all stochastic terms except for photons to predict the photon-limited LWR for six resist achieving $16-\mathrm{nm}$ half pitch resolution or better, four chemically amplified (CA) and two not (NCA). Table 1 shows a summary of the results including the measured resolution limit, measured sensitivity, extracted blur based on measured LWR correlation length, predicted photon limited LWR, and DTR based on a target LWR of $1.2 \mathrm{~nm}$. The target LWR value is taken from the ITRS [9] for the 16-nm M1 half-pitch node in the year 2021.

The results show that for all CA resists, the current absorptivity is expected to limit us to a minimum dose of approximately $90 \mathrm{~mJ} / \mathrm{cm}^{2}$ in order to achieve the target LWR of $1.2 \mathrm{~nm}$. For NCA resists, on the other hand, although they are currently significantly slower than CA resist, their current absorptivity yields an expected dose limit down to $45 \mathrm{~mJ} / \mathrm{cm}^{2}$. So we see that key to progress in CA resists is to increase absorptivity whereas key to progress in NCA resists is to increase the amount of chemistry done by each absorbed photon.
Table 1. Predicted photon limited LWR performance and DTR for six resists with 16-nm or better half-pitch resolution.

\begin{tabular}{|c|c|c|c|c|c|c|}
\hline & $\begin{array}{c}\text { Resist } \\
\text { CA-A }\end{array}$ & $\begin{array}{c}\text { Resist } \\
\text { CA-B }\end{array}$ & $\begin{array}{c}\text { Resist } \\
\text { CA-C }\end{array}$ & $\begin{array}{c}\text { Resist } \\
\text { NCA-A }\end{array}$ & $\begin{array}{c}\text { Resist } \\
\text { CA-D }\end{array}$ & $\begin{array}{c}\text { Resist } \\
\text { NCA-B }\end{array}$ \\
\hline $\begin{array}{c}\text { Resolution } \\
(\mathrm{nm})\end{array}$ & $\mathbf{1 6}$ & $\mathbf{1 6}$ & $\mathbf{1 5}$ & $\mathbf{1 5}$ & $\mathbf{1 3}$ & $\mathbf{1 3}$ \\
\hline $\begin{array}{c}\text { Sensitivity } \\
\left(\mathrm{mJ} / \mathrm{cm}^{2}\right)\end{array}$ & $\mathbf{3 0}$ & $\mathbf{2 0}$ & $\mathbf{2 2}$ & $\mathbf{8 0}$ & $\mathbf{3 5}$ & $\mathbf{6 0}$ \\
\hline Blur $(\mathrm{nm})$ & $\mathbf{1 2}$ & $\mathbf{1 1}$ & $\mathbf{1 0}$ & $\mathbf{7}$ & $\mathbf{1 0}$ & $\mathbf{1 2}$ \\
\hline $\begin{array}{c}\text { Photon } \\
\text { LWR }(\mathrm{nm})\end{array}$ & $\mathbf{2 . 1}$ & $\mathbf{2 . 7}$ & $\mathbf{2 . 5}$ & $\mathbf{0 . 9}$ & $\mathbf{1 . 9}$ & 1.2 \\
\hline $\begin{array}{c}\text { DTR } \\
\left(\mathrm{mJ} / \mathrm{cm}^{2}\right)\end{array}$ & $\mathbf{9 2}$ & $\mathbf{1 0 1}$ & $\mathbf{9 5}$ & $\mathbf{4 5}$ & $\mathbf{8 8}$ & $\mathbf{6 0}$ \\
\hline
\end{tabular}

For reference, in Table 2 we further show the measured LWR and estimated material LWR based on the quadrature subtraction of the estimated photon LWR from the measured LWR. In all cases except for the NCA-A resist, we find the residual material LWR to significantly exceed target specifications. This indicates that significant progress is also required on the materials stochastics.

Table 2. Measured LWR and estimated residual material LWR based on quadrature subtraction of estimated photon LWR.

\begin{tabular}{|c|c|c|c|c|c|c|} 
& $\begin{array}{c}\text { Resist } \\
\text { CA-A }\end{array}$ & $\begin{array}{c}\text { Resist } \\
\text { CA-B }\end{array}$ & $\begin{array}{c}\text { Resist } \\
\text { CA-C }\end{array}$ & $\begin{array}{c}\text { Resist } \\
\text { NCA-A }\end{array}$ & $\begin{array}{c}\text { Resist } \\
\text { CA-D }\end{array}$ & $\begin{array}{c}\text { Resist } \\
\text { NCA-B }\end{array}$ \\
\cline { 2 - 7 } $\begin{array}{c}\text { Resolution } \\
(\mathrm{nm})\end{array}$ & $\mathbf{1 6}$ & $\mathbf{1 6}$ & 15 & 15 & 13 & 13 \\
\hline LWR $(\mathrm{nm})$ & 3.1 & 4.8 & 3.8 & 1.5 & 3.5 & 3.1 \\
\cline { 2 - 7 } $\begin{array}{c}\text { Photon } \\
\text { LWR }(\mathrm{nm})\end{array}$ & 2.1 & 2.7 & 2.5 & 0.9 & 1.8 & 1.2 \\
\hline $\begin{array}{c}\text { Material } \\
\text { LWR }(\mathrm{nm})\end{array}$ & 2.3 & 4.0 & 2.9 & 1.2 & 2.9 & 2.8 \\
\hline
\end{tabular}

\section{High efficiency contact hole patterning}

Contact hole patterning is expected to be one of the first high volume applications for EUV lithography and is a significant driver in shot noise requirements. The fact that conventional contact hole patterning typically requires at least $2 \times$ the dose compared to line-space patterning further exacerbates the concern over shot noise limits and resist sensitivity. The loss of efficiency relative to line-space patterning, however, is not a resist effect, but rather an optical effect.

As previously described, the contact 
efficiency problem can be mitigated by way of mask design [1,2] with a checkerboard phase shift design being the most effective. Using such methods, simple thin mask modeling predicts throughput gains on the order of $4 \mathrm{X}$ compared to a conventional darkfield contact hole mask. Further considering 3D effects, actual throughput gains are expected to be even higher.

Figure 4 shows the checkerboard phase shift mask concept. The mask is comprised of a checkboard pattern which when printed yields a regular grid of contacts where the printed CD further benefits from pitch splitting in addition to the lithographic demagnification.

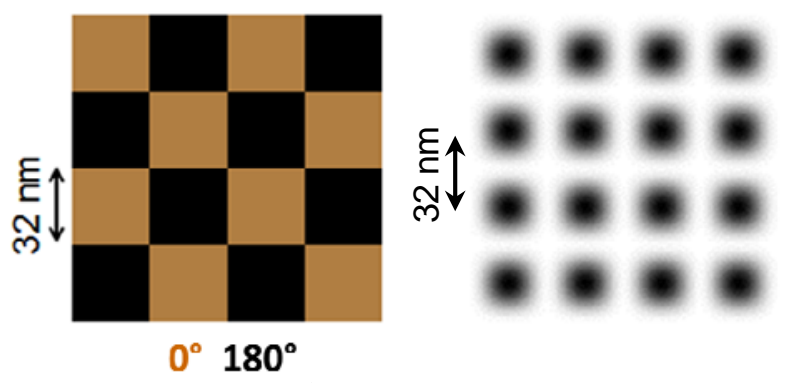

Figure 4. Phase only checkerboard design (left) which prints (right) a pitch split square grid of contacts.

The etched multilayer phase shift mask fabrication process and preliminary scatterometry characterization of a line-space test mask has been described in the literature [2]. Figure 5 shows a scanning electron microscope image of contacts coded to print as 32-nm half pitch on a 4 reduction system.

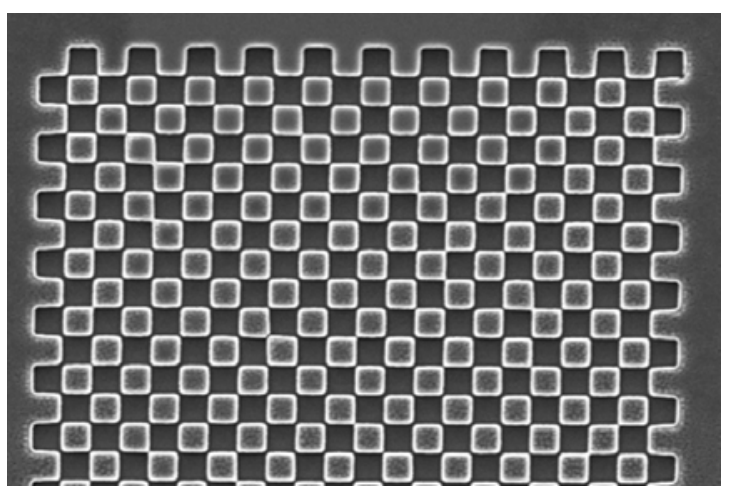

Figure 5. Scanning electron microscope image from phase shift mask of contacts coded to print as $32-n m$ half pitch on a $4 \times$ reduction system.
To facilitate development, the test phase shift mask has been fabricated onto a wafer instead of a conventional mask substrate. Since this substrate significantly complicates printing in the EUV microfield exposure tool in Berkeley [10,11], initial patterning and throughput characterization is performed using the SHARP EUV microscope at Berkeley [12] to mimic a $4 \mathrm{X}$ magnification exposure tool with wafer side numerical aperture of 0.33 . Figure 6 shows a direct comparison of $25-\mathrm{nm}$ half pitch contact imaging for the phase shift mask and conventional mask with identical dose conditions at the mask. The improved efficiency is readily evident through the observed difference in photon noise (pattern variability).
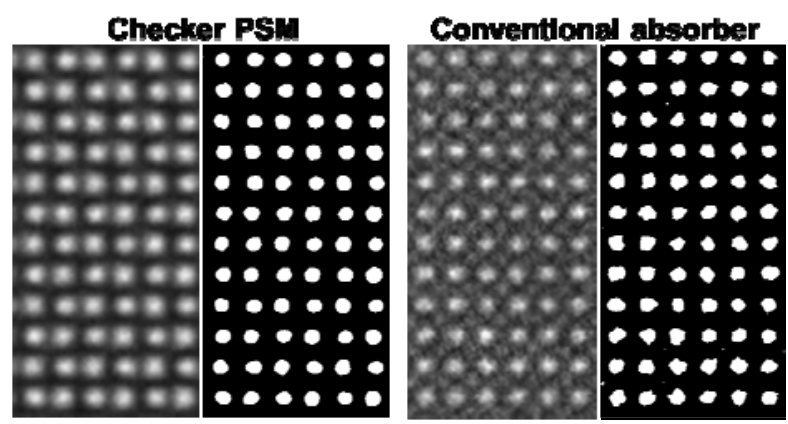

Figure 6. Direct imaging comparison in SHARP microscope of checkerboard PSM and conventional absorber masks for $25-n m$ contacts.

We quantify the throughput gain afforded by the checkerboard PSM mask by plotting the $C D$ as a function of exposure level (Figure 7). The results show a throughput increase of $8 x$ and an approximate $2 x$ reduction in horizontal vertical bias. Repeating the same measurements for 32-nm contacts, we find a throughput increase of $4.6 \mathrm{X}$ and an approximate $1.5 \mathrm{X}$ reduction horizontal vertical bias.

\section{Summary}

The concept of DTR has been introduced showing that absorptivity is expected to limit current CA resist to a minimum dose of $\sim 90 \mathrm{~mJ} / \mathrm{cm}^{2}$ and current NCA resists to a minimum dose of $\sim 45 \mathrm{~mJ} / \mathrm{cm}^{2}$. Also, in most cases we find current resist LWR performance to be limited by residual material terms as opposed to absorbed photon limits.

We have also demonstrated the use of a 
checkerboard PSM for dramatic optical efficiency optimization. Using this method, we have shown throughput increases compared to conventional absorber masks of $8 \mathrm{x}$ and $4.6 \mathrm{x}$ for contact half pitches of $25 \mathrm{~nm}$ and $32 \mathrm{~nm}$, respectively.
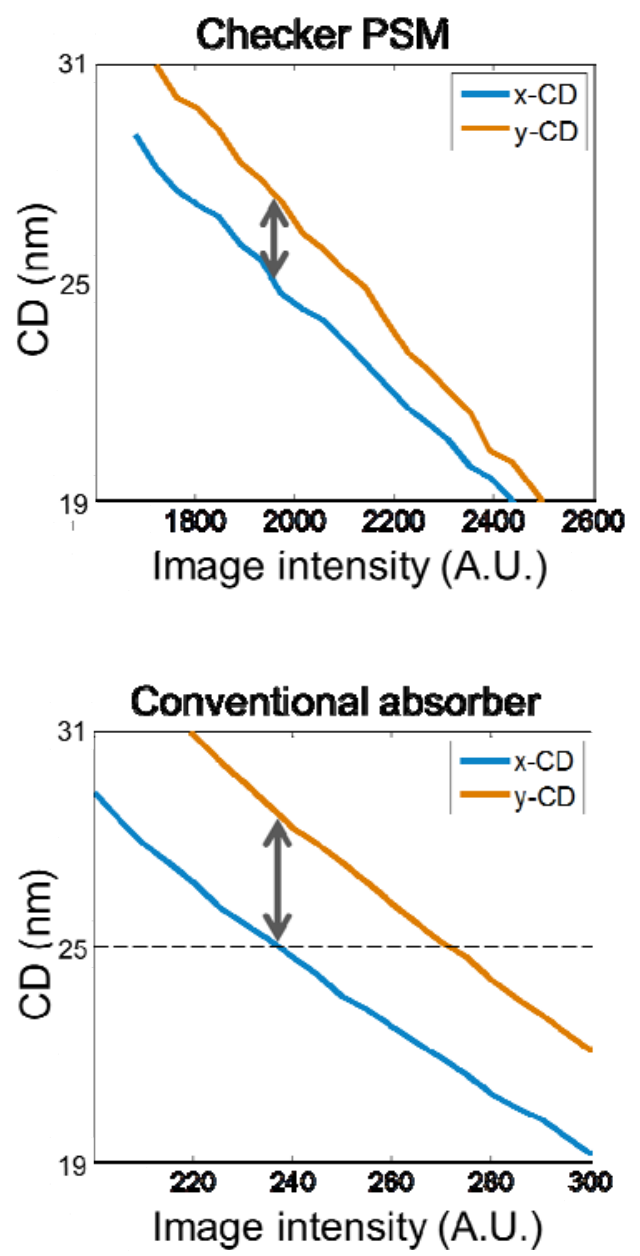

Figure 7. Direct comparison of $\mathrm{CD}$ as a function of exposure level for the checkerboard PSM and conventional absorber masks on 25-nm contacts.

\section{Acknowledgements}

The authors are greatly indebted to the CXRO MET operations team including Gideon Jones, Mark Binenbaum, Chanin King, Kurt Schlueter, and Jessalyn Sincher. We also thank Rama Ayothi of JSR, Hideaki Tsubaki of Fuji, and Jason Stowers of Inpria for excellent resist support. The work was performed at Lawrence Berkeley National Laboratory's Advanced Light Source synchrotron facility with support from Inpria, Intel, and JSR through the U.S. Department of Energy under Contract No. DE-AC02-05CH11231.

\section{References}

1. P. Naulleau, C. Anderson, S. Bhattarai, A. Neureuther, "EUV extendibility: challenges facing EUV at 1x and beyond", J. Photopolym. Sci. Technol., 26, 697 (2013).

2. P. Naulleau, C. Anderson, W. Chao, S. Bhattarai, A. Neureuther, K. Cummings, S. Jen, M. Neisser, B. Thomas "EUV resists: pushing to the extreme”, J. Photopolym. Sci. Technol., 27, 725-730 (2014).

3. G. Gallatin, P. Naulleau, R. Brainard, "Fundamental Limits to EUV Photoresist", Proc. SPIE 6519, 651911 (2007).

4. T. Wallow, C. Higgins, R. Brainard, K. Petrillo, W. Montgomery, C. Koay, G. Denbeaux, O. Wood, Y. Wei, "Evaluation of EUV resist materials for use at the $32 \mathrm{~nm}$ half-pitch node", Proc. SPIE 6921, 69211F (2008).

5. G. Gallatin, "Resist blur and line edge roughness”, Proc. SPIE 5754, 38 (2005).

6. P. Naulleau and G. Gallatin, "The effect of resist on the transfer of line-edge roughness spatial metrics from mask to wafer", J. Vac. Sci. \& Technol. B, 28, 1259 (2010).

7. Stochastic modeling and LWR correlation length measurements performed using SuMMIT LER analysis software (www.lithometrix.com).

8. C. Anderson and P. Naulleau, "A high-throughput contact-hole resolution metric for photoresists: full-process sensitivity study", Proc. SPIE 6923, 69230Z (2008).

9. http://www.itrs.net/Links/2013ITRS/ Summary2013.htm

10. P. Naulleau, K. A. Goldberg, E. H. Anderson, K. Bradley, R. Delano, P. Denham, B. Gunion, B. Harteneck, B. Hoef, H. Huang, K. Jackson, G. Jones, D. Kemp, J. A. Liddle, R. Oort, A. Rawlins, S. Rekawa, F. Salmassi, R. Tackaberry, C. Chung, L. Hale, D. Phillion, G. Sommargren, J. Taylor, "Status of EUV microexposure capabilities at the ALS using the 0.3-NA MET optic", Proc. SPIE 5374, 881 (2004).

11. C. Anderson, D. Ashworth, L. M. Baclea-An, S. Bhattari, R. Chao, R. Claus, P. Denham, K. Goldberg, A. Grenville, G. Jones, R. Miyakawa, K. Murayama, H. Nakagawa, S. Rekawa, J. Stowers, P. Naulleau, "The SEMATECH Berkeley MET: demonstration of 15-nm half-pitch in chemically amplified EUV resist and sensitivity of EUV resists at 6.x-nm”, Proc. SPIE 8322, 832212 (2012). 
12. K. A. Goldberg, I. Mochi, M. P. Benk, C. Lin, A. Allezy, M. Dickinson, C. W. Cork, J. B. Macdougall, E. H. Anderson, W. Chao, F. Salmassi, E. M. Gullikson, D. Zehm, V. Vytla, W. Cork, J. DePonte, G. Picchi, A. Pekedis, T. Katayanagi, M. G. Jones, E. Martin, P. P.
Naulleau, S. B. Rekawa, "The SEMATECH high-NA actinic reticle review project (SHARP) EUV mask-imaging microscope”, Proc. SPIE 8880, 88800T; doi: 10.1117/12.2026496 (2013). 